\title{
Analysis strategy issues for the maintenance of the ICRF axes ${ }^{\star}$
}

\author{
M. Feissel-Vernier ${ }^{1}$, C. $\mathrm{Ma}^{2}$, A.-M. Gontier ${ }^{1}$, and C. Barache ${ }^{1}$ \\ 1 Observatoire de Paris/SYRTE, CNRS UMR8630, 61 Av. de l'Observatoire, 75014, Paris, France \\ e-mail: mf.vernier@gmail.com; [anne-marie.gontier; christophe.barache]@obspm. fr \\ 2 NASA/Goddard Space Flight Center, USA \\ e-mail: chopo.ma@nasa.gov
}

Received 24 November 2005 / Accepted 27 January 2006

\section{ABSTRACT}

\begin{abstract}
In preparation for the evolution of the definition of the VLBI-based International Celestial Reference Frame (ICRF), various elements of the analysis strategy are investigated, such as reference source selection and the impact of the status of the terrestrial reference frame in the data analysis model. We conclude that including the determination of both the celestial and the terrestrial reference frames in the analysis does not affect the quality of the celestial reference frame. The determination of precession and nutation components is not affected by the status of the terrestrial reference frame but it is affected by the selection of reference radio sources. Extending an earlier study, three lists of reference sources based on progressively larger tolerances are proposed. They include 181,225 and 247 objects, respectively.
\end{abstract}

Key words. astrometry - reference systems - galaxies: quasars: general

\section{Introduction}

The International Celestial Reference Frame (ICRF) was derived by Ma et al. (1998) from the VLBI observation of extragalactic radio sources acquired until the middle of 1995. Two successive extensions have been issued since then (Fey et al. 2004), which kept the core of the ICRF computation unchanged and provided updated or new coordinates for some of the radio sources. The general improvements in VLBI technology, development of the observing network, and extension of the set of observed objects that took place in operational VLBI, all brought the astrometric results to the precision embodied in the current ICRF towards the end of the first decade of operation. Starting about 1990, the individual time-series of source coordinates stabilized (Gontier et al. 2003).

Several studies have shown that this progress in data quality and in the understanding of the behaviour of the radio sources would justify revisiting the ICRF calculation procedures. In a study of individual source motions, Feissel-Vernier (2003, referred to as MFV in the rest of the paper) devised a stability selection scheme that pinpointed 163 well observed sources that are highly unstable or drifting in the 1990-2002 time frame. Conversely, a set of 199 well-observed and stable sources was identified. Another set of 358 sources was observed too sparsely in this time frame for a statistical evaluation of their stability. Using the stable sources in the definition of the axes of a celestial reference frame was shown to lower the medium-term instability of the celestial reference frame from 28 to $6 \mu$ as when compared to the ICRF. Background information on this selection process is given by Feissel-Vernier (2006). It was shown that VLBI results based on this source selection are more consistent than those obtained in the conventional manner (Dehant et al. 2003; Gontier \& Feissel-Vernier 2003; Arias \& Bouquillon 2004;

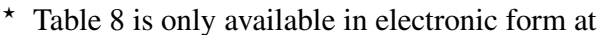
http: //WwW .edpsciences.org
Feissel-Vernier et al. 2005). In summary, the core of stable sources allows a better match of various celestial reference frames, each of which is attached to the ICRF in a slightly different manner. Orienting the celestial frame using the stable sources improves the precision of the source positions by 10 to $30 \%$ when compared to the conventional use of the ICRF defining sources. In addition, the set of selected sources influences the determination of precession and the principal term of nutation at the level of tens of microarcseconds ( $\mu$ as), i.e. comparable to the level of the geophysical modelling uncertainties. The derived time-series of pole coordinates and universal time are stabler when the unstable sources are not included in the reference frame.

Since the publication of the initial ICRF, additional, albeit incomplete, observations of the radio sources have been carried out by the International VLBI Service for geodesy and astrometry (IVS), extending by a large amount the good quality data available. Beginning in early 2004 a systematic observing program for stable and potentially stable sources was begun using some of the regularly scheduled geodetic sessions. The goal is to observe each such source, as well as all ICRF defining sources, at least once every six months. A separate astrometric observing program for the southern hemisphere has been in place for several years. Figures 1-4 show the observational histories of the ICRF defining sources, of the stable and unstable sources according to the MFV study, and of the remaining, sparsely observed sources, respectively. Each dot represents a session in which a source with a given declination was observed.

In Sect. 2, we recall the principles of the MFV selection scheme and we study its performance over the pre-1995 data that were available to the authors of the ICRF. In Sect. 3 we identify a set of additional stable sources and the sparsely observed sources that could be expected to be stable. In Sect. 4 we discuss the impact of the analysis strategy concerning the station coordinates on the celestial reference frame consistency 


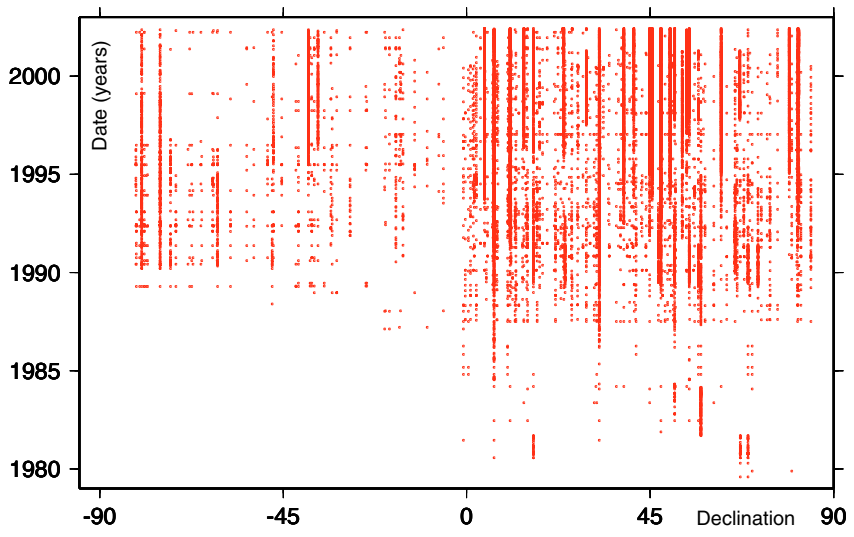

Fig. 1. 1979-2002 observations of the 212 ICRF defining sources.

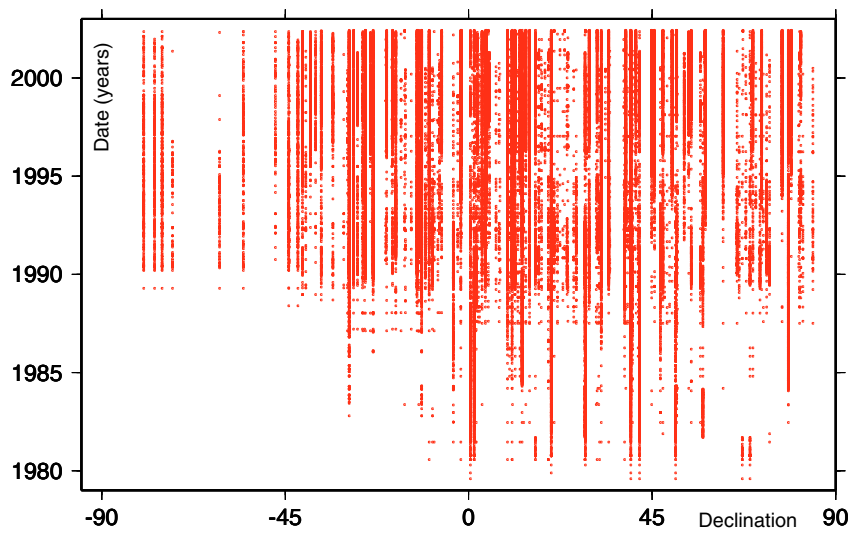

Fig. 2. 1979-2002 observations of 199 stable sources according to MFV.

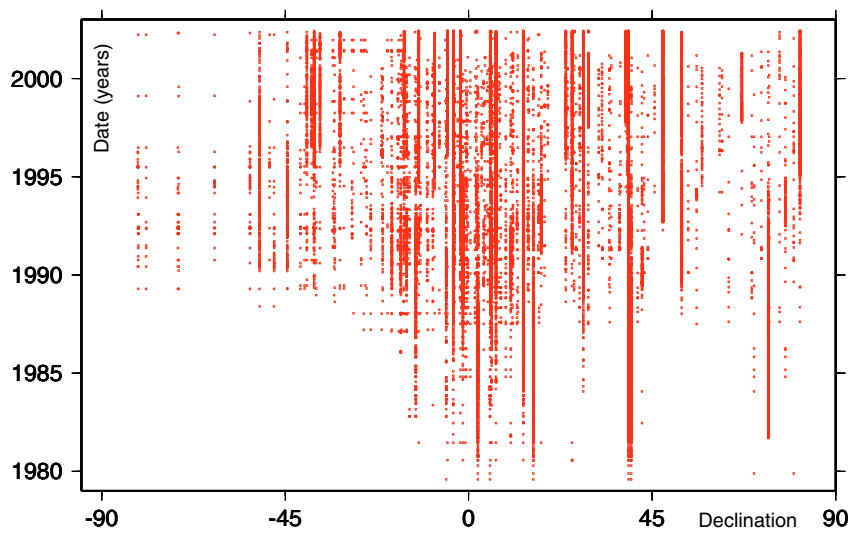

Fig. 3. 1979-2002 observations of 163 unstable sources according to MFV.

and the derived precession and nutation parameters. Finally we summarise in Sect. 5 the arguments in favour of new analysis conditions when the ICRF is revised.

\section{Investigation of the radio source stability}

Feissel-Vernier (2003) mentioned the relatively poor correlation of the results of her statistical selection of unstable and stable sources with the ICRF quality indices: defining/candidate/other categories and source structure index. To get a clearer view of the respective performances of the ICRF classification and of the MFV stability-instability recognition scheme, the same process was applied to the series of source coordinates over the time

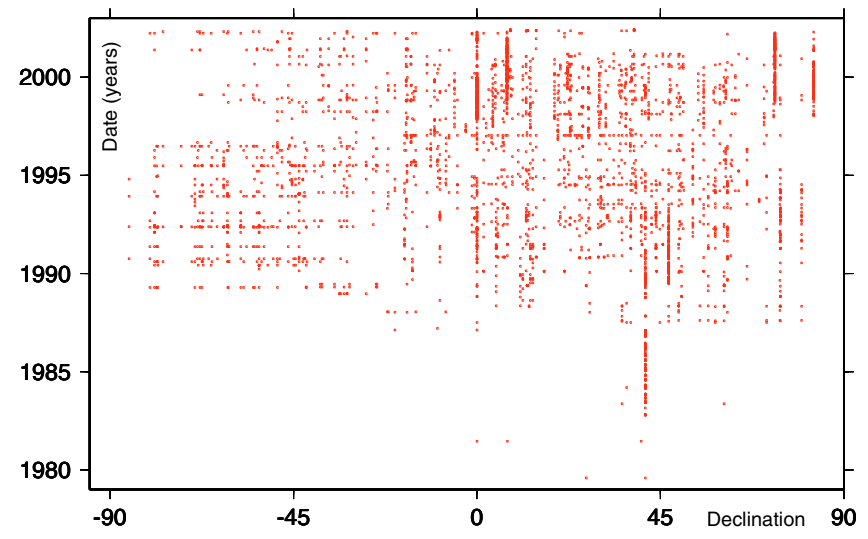

Fig. 4. 1979-2002 observations of the sparsely observed sources.

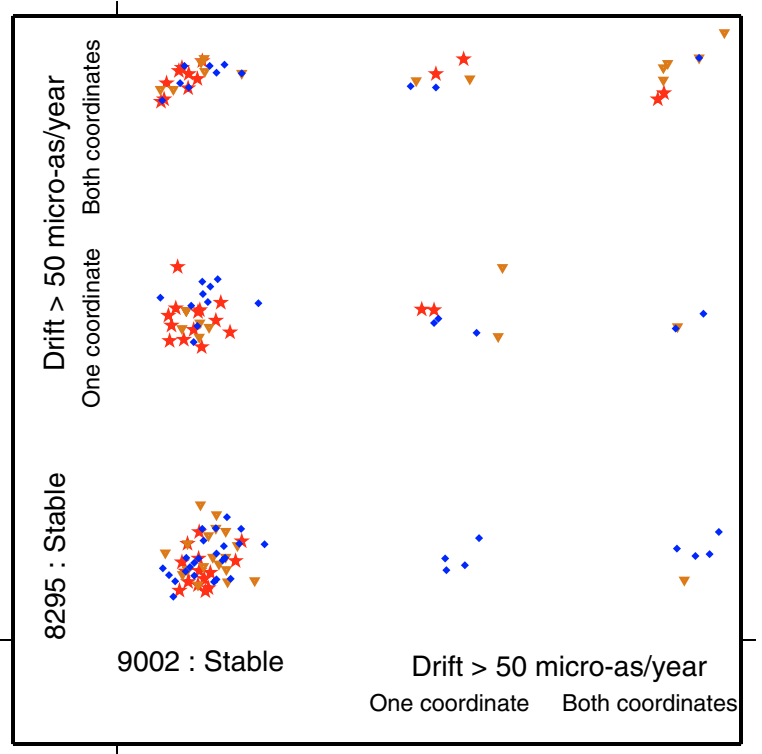

Fig. 5. Criteria comparison: measured stability 1982-1995 (vertical scale) vs. 1990-2002 (horizontal scale) for the 143 sources that were well-observed in both time spans. Key to the ICRF source status: defining (red stars), candidate (yellow triangles), other (blue diamonds).

frame that was available to the ICRF authors, i.e. 1980-1995. Among the 151 sources with sufficient data for analysis, 65 were found stable.

The comparison of source qualifiers is shown graphically in Fig. 5 for the 143 common sources that were observed enough over the two time frames 1980-1995 and 1990-2002. These include 43 defining sources, 42 candidates, and 58 other. The sources appear in clusters corresponding to stable sources (bottom and left), drifting or highly unstable in one coordinate (centre) or in both coordinates (up and right). The ICRF status is also shown: defining sources by stars, candidate sources by triangles, and other sources by diamonds. If the results of the stability recognition scheme applied in the two time frames were the same, the clusters would be aligned on the diagonal of the frame. While this is the case for those sources that are found stable in both time frames, a remarkable result is that many sources that are found stable in the recent data (1990-2002) are found to be unstable in the earlier ones (1982-1995). Those include defining sources as well as candidates or other sources.

If the MFV stability test had be applied at the time of the ICRF publication, many sources considered then as not known well enough to be used for defining the direction of the ICRF 
axes would have been selected, and conversely a number of defining sources would not have received this status.

\section{Extending the list of stable sources}

The MFV stability detection scheme is based on relatively strict thresholds, concerning in particular the amount of observations available. A selection scheme based on less complete statistics, described in Sect. 3.1, allows us to identify 22 sources that are likely to be stable. Section 3.3 presents a "second generation" detection of stable sources, based on a slightly modified scheme.

\subsection{Further investigation of the sparsely observed sources}

The MFV stability detection is based on time-series statistics of yearly averaged source coordinates. It has therefore the limitation of requiring a time-density of observation. Over the 1990-2002 time span, 358 sources, i.e. about half of the total, were considered too sparsely observed to be submitted to the scheme. In order to learn more about their possible stability, a simplified selection scheme was applied to those sources. For sources observed in more than 3 sessions over more than 2 years, we assume that a standard deviation of the weighted mean position smaller than 0.5 mas in both $\alpha \cos \delta$ and $\delta$ and a linear drift smaller than $50 \mu$ as/year in both $\alpha \cos \delta$ and $\delta$ are an indication that the source might be stable. We thus select a set of 22 additional sources.

\subsection{Second generation stable sources}

The MFV scheme was applied to the time-series of source coordinates that were derived taking subsets of the ICRF defining sources as the background reference. As a sizeable part of those were detected as unstable, one could not rule out the possibility that the intrinsic instability of the set of reference sources create spurious instabilities in the sources under study. A slightly modified scheme was therefore applied to the time-series of source coordinates derived from a global analysis where the original 199 stable sources were held fixed relative to the ICRF through a no-net-rotation (NNR) condition, using the CALC-SOLVE software package. An additional rejection condition was introduced: apparent drifts in $\alpha \cos \delta$ or $\delta$ larger than $10 \mu \mathrm{as} / \mathrm{year}$, at a $5-\sigma$ significance. A set of 44 sources were detected as stable. In this process, the following nine sources were rejected by the additional instability condition: $0014+813,0248+430,0722+145,0859+470,0923+392$ (4C 39.25), 0955+476, 2007+777, 2029+121, 2121+053. The source names used here are the IERS (2004) source designations based on the B1950 coordinates. The correspondence with names used by the IVS are given in brackets.

When the additional instability condition is applied to the time-series used by Feissel-Vernier (2003), the following 18 sources are found to be unstable. $0059+581,0202+149$, $0229+131, \quad 0234+285, \quad 0602+673, \quad 0738+313, \quad 0745+241$, 0749+540, 1045-188, 1053+704, 1404+286 (OQ208), $1502+106,1606+106,1611+343,1638+398$ (NRAO512), $1642+690,1726+455,1738+476$. This modified scheme reduces the original set of 199 stable sources to only 181 .

\subsection{Enhanced set of stable sources}

The extension and revision of the original Feissel-Vernier (2003) source selection may be summarised as follows.
Table 1. Correspondence between IERS and IVS source names, when different.

\begin{tabular}{ll|ll}
\hline \hline IERS & IVS & IERS & IVS \\
\hline $0430+052$ & 3 C 120 & $1744+557$ & NGC6454 \\
$1222+131$ & M84 & $1901+319$ & 3 C395 \\
$1458+718$ & 3 C 309.1 & $2017+745$ & $2017+743$ \\
$1641+399$ & 3 C345 & & \\
\hline
\end{tabular}

- Adding the exclusion of sources with apparent drifts in $\alpha$ $\cos \delta$ or $\delta$ larger than $10 \mu$ as/year with a 5- $\sigma$ significance level to the MFV stability scheme, and applying it to the data used by Feissel-Vernier (2003) provides a first list of 181 proposed reference sources.

- Selecting sources with low position scatter in the sparsely observed sources over 1990-2002 (Sect. 3.1) adds another 27 objects to the proposed reference sources.

- Applying the modified stability scheme to the time-series of source coordinates derived from a global analysis where the original 199 stable sources where held fixed (Sect. 3.3) provides 44 additional proposed reference sources.

Table 8 lists the 247 sources that are thus considered suitable for the maintenance of the ICRF axes. Notes to Table 8 are the following.

1. IERS source designations. The correspondence with names used by the IVS, when different, is given in Table 1.

2. Type of Object: Q: quasar; G: galaxy; L: BL Lac; A: other.

3. ">" left of the value means that the latter is a lower limit.

4. "*" indicates that the reported value is the flux at $11 \mathrm{~cm}$, rather than $15 \mathrm{~cm}$.

5. Structure index at $\mathrm{X}$-band (X) and S-band (S). This index (Fey \& Charlot 2000) qualifies the level of position disturbance expected as a result of the the source structure (1 for the least disturbed, 4 for the most disturbed).

6. Category of stability detection step. 1: MFV2003 stable sources with an additional rejection condition (181 objects); 2: simplified selection scheme for sparsely observed sources (22 objects); 3: stable sources against the MFV2003 stable sources background, using the additional rejection condition (44 objects).

Information for notes 1 and 5 is taken from Fey et al. (2004). Information for notes 2, 3, and 4 is taken from ICRS-PC (2004).

\section{Sensitivity of the celestial reference frame to the terrestrial reference frame status in the data analysis}

The analysis strategy for deriving a celestial reference frame from multi-year VLBI observations includes a number of choices. We consider here the choice that has to be made concerning the definition of the celestial and terrestrial reference frames and their connection in time. Two different approaches are used, as follows.

- In the derivation of the ICRF and its extensions, the so-called CRF approach was used, namely the station positions are set as arc parameters; i.e., they are estimated independently for each observing session. Polar motion and universal time are not estimated but nutation corrections (celestial pole offsets) are obtained. This strategy was chosen in order to free 
Table 2. Test TRF and CRF solutions: status of the sources (Global or Arc) and category of sources considered to define the orientation of the celestial frame (No-net-rotation).

\begin{tabular}{|c|c|c|c|}
\hline Frame & Arc sources & Global sources & No-net-rotation \\
\hline \multicolumn{4}{|c|}{ TRF approach } \\
\hline cne & unstable & all others & stable \\
\hline$c n 7$ & none & all & ICRF defining \\
\hline \multicolumn{4}{|c|}{ CRF approach } \\
\hline$c n h$ & unstable & all others & stable \\
\hline cn8 & none & all & ICRF defining \\
\hline
\end{tabular}

the celestial frame solution from systematic errors that may propagate from terrestrial network deficiencies.

- In the so-called TRF approach, most station positions and velocities are set as global parameters, i.e. considered as valid over the total data span. Polar motion, universal time, and nutation corrections are estimated for each session.

We investigated this possible contamination using test solutions based on the 1980.0-2002.7 data, described in Table 2. The results considered are the celestial reference frames on the one hand, and the time-series of celestial pole offsets on the other hand. Data analysis was performed with the CALC-SOLVE software package.

\subsection{Impact on the orientation of the celestial reference frame}

In order to test the possible perturbation of the orientation of the celestial reference frame due to considering a global terrestrial frame, i.e. one set of station positions and velocities, we computed the relative orientations of pairs of celestial frames obtained with the same source categorisation, using either the TRF or the CRF approach.

The relative orientation of two celestial reference frames is modelled by three rotation angles $A_{1}, A_{2}, A_{3}$ around the axes of the equatorial coordinate system. These angles are estimated using Eqs. (1) and (2), where $\alpha, \delta$ are the source coordinates and $\Delta \alpha, \Delta \delta$ are the differences of coordinates in the two frames:

$\Delta \alpha=A_{1} \tan \delta \cos \alpha+A_{2} \tan \delta \sin \alpha-A_{3}$

$\Delta \delta=-A_{1} \sin \alpha+A_{2} \cos \alpha+\mathrm{d} z$

The $\mathrm{d} z$ parameter is a fictitious declination bias that reflects systematic differences in declination that may be caused, e.g., by inaccuracy of the tropospheric propagation correction for sources observed at low elevations. This is often the case for sources in the equatorial region, as a result of the terrestrial network geometry. In the pre-ICRF celestial frames, significant values of $d z$ were found when comparing solutions obtained by different analysts. However, McMillan \& Ma (1997) showed that adding the so-called gradient parameter in the modelling of the tropospheric delay minimises this defect. The gradient parametrisation is now commonly used in VLBI analysis. Although the $\mathrm{d} z$ parameter is expected to be negligible, it is still written in the model to satisfy the requirements of the least-square estimation. This offers an additional quality check.

The relative angles between pairs of celestial reference frames are given in Table 3, for the two pairs of celestial frames described in Table 2. In the case of the conventional source selection ( $c n 7$ and $c n 8)$, the inconsistency of the axes definition between the TRF and CRF approaches is smaller than $7 \mu$ as, which is well below the published accuracy of the ICRF axes
Table 3. Relative rotation angles and equator tilt of celestial reference frames obtained with the TRF and $\mathbf{C R F}$ approaches, respectively. Unit: $\mu$ as.

\begin{tabular}{lcccc}
\hline \hline Pair & \multicolumn{1}{c}{$A_{1}$} & $A_{2}$ & $A_{3}$ & $d z$ \\
\hline Reference: Stable sources & & & \\
cne-cnh & $0.7 \pm 0.6$ & $0.2 \pm 0.6$ & $-2.3 \pm 0.6$ & $0.5 \pm 0.9$ \\
Reference: Defining sources & & & \\
cn7-cn8 & $-6.9 \pm 1.0$ & $6.6 \pm 1.0$ & $1.7 \pm 1.4$ & $-4.2 \pm 1.4$ \\
\hline
\end{tabular}

(20 $\mu$ as). In the case of the selection of stable sources (cne and cnh), it is even smaller (less than $3 \mu$ as).

\subsection{Impact on the precision of the celestial reference frame}

To evaluate the impact of the TRF approach on the precision of the source positions, we compare them with two independent celestial frames available at the IERS/ICRS Product Center (ICRS-PC 2003), one provided by the IAA (St Petersburg) and the other by BKG (Leipzig). Note that the cne and $c n h$ frames of Table 2 were aligned onto the ICRS axes using a no-net-rotation condition based on the 199 stable sources, while for $c n 7, c n 8$ and the two independent frames, the NNR condition was based on the 212 ICRF defining sources. The analysis strategy used to derive the independent celestial reference frames follows the TRF approach.

In each comparison, the two frames are rotated to common axes in a least-square adjustment of Eqs. (1) and (2) applied to the 206 sources that are common to the six reference frames. These are by definition global sources. As in the construction of two of the compared frames, cne and cnh, the unstable sources were Arc sources. There are no unstable sources in the common list. About $2 / 3$ of the common sources belong to the stable category, the other $1 / 3$ being sparsely observed.

The variances of the post fit $\Delta \alpha \cos \delta$ and $\Delta \delta$ residuals are computed. In this process, the estimated parameters $A_{1}, A_{2}, A_{3}$, and $\mathrm{d} z$ absorb systematic differences that can be expected from the construction schemes of the compared reference frames. The variance of the residuals is a measure of the residual differences.

The three-cornered-hat method is then applied to these variances. This method allows estimation of the variance of the noises of three or more sets of measurements of the same quantities, here the source right ascensions and declinations, under the assumption that the errors are independent in the compared data sets. Considering the variances of the differences between two data sets $i$ and $j$, one can write

$\operatorname{var}\left(\alpha_{i} \cos \alpha-\alpha_{j} \cos \alpha\right)=\operatorname{var}\left(\alpha_{i} \cos \alpha\right)+\operatorname{var}\left(\alpha_{j} \cos \alpha\right)$

$\operatorname{var}\left(\delta_{i}-\delta_{j}\right)=\operatorname{var}\left(\delta_{i}\right)+\operatorname{var}\left(\delta_{j}\right)$

If three reference frames are considered, one gets three sets of the above equations, that can be solved to obtain the individual residual variances $\operatorname{var}\left(\alpha_{k} \cos \alpha\right)$ and $\operatorname{var}\left(\delta_{k}\right)$ for $k=1,3$. If more than three data sets with independent errors are available, the individual residual variances can be estimated by means of a statistical algorithm, e.g. by least-square or L1 Norm estimation.

Table 4 gives the individual standard deviations thus derived for the reference frames of Table 2. The direct comparison of the four frames under study was indeed not used in this process, as their errors cannot be assumed to be independent. The robustness of the estimations was tested by associating and analysing the data in various ways. The results remain stable within $2 \mu$ as. 
Table 4. Standard deviations of source positions for celestial reference frames derived by the TRF and CRF approaches. Unit: $\mu$ as.

\begin{tabular}{ccccccc}
\hline \hline Source & \multicolumn{3}{c}{ TRF approach } & \multicolumn{3}{c}{ CRF approach } \\
selection & \multicolumn{2}{c}{ Standard deviations } & \multicolumn{3}{c}{ Standard deviations } \\
& sol. & $\Delta \alpha \cos \delta$ & $\Delta \delta$ & sol. & $\Delta \alpha \cos \delta$ & $\Delta \delta$ \\
\hline Proposed & cne & 27.1 & 21.2 & cnh & 28.0 & 21.1 \\
Convent. & cn7 & 28.2 & 27.5 & cn8 & 29.4 & 24.7 \\
\hline
\end{tabular}

Table 5. Precession and obliquity rate corrections: role of the terrestrial reference frame status and of the source selection.

\begin{tabular}{lcc}
\hline \hline \multicolumn{1}{c}{$\begin{array}{c}\text { Source } \\
\text { selection }\end{array}$} & $\begin{array}{c}\text { Precession } \\
\mu \text { as/year }\end{array}$ & $\begin{array}{c}\text { Obliqu. rate } \\
\mu \text { as/year }\end{array}$ \\
\hline TRF approach & & \\
Proposed $(c n e)$ & $-56.3 \pm 4.8$ & $-31.6 \pm 1.8$ \\
Conventional $(c n 7)$ & $-17.6 \pm 4.2$ & $-28.5 \pm 1.7$ \\
& & \\
CRF approach & & \\
Proposed $(c n h)$ & $-58.2 \pm 4.6$ & $-32.4 \pm 1.7$ \\
Conventional $(c n 8)$ & $-15.4 \pm 4.0$ & $-27.0 \pm 1.6$ \\
\hline
\end{tabular}

In the case of the conventional source selection, as well as in that of the selection of stable sources, we conclude from the values of Table 4 that the precision of the selected source positions is better that $30 \mu$ as and that the impact of the TRF status in the data analysis is smaller than $10 \%$ of this value.

\subsection{Impact on the derived precession and nutation}

The computation of the test celestial frames included the estimation of the celestial pole offsets $\Delta \psi$ and $\Delta \epsilon$ for each session, considering only the global sources. These time-series were compared to the prediction of the IAU2000 nutation model. Note that the latter was obtained by Mathews et al. (2002) starting from an analysis of VLBI observations where all sources were considered global, a source selection scheme analogous to that leading to celestial frame $c n 7$, and using the TRF approach.

The comparison aims at showing the size of the differences of estimates of the low frequency components of the celestial pole motion. It is performed over the 1984.0-2002.7 time span. The parameters investigated are precession and obliquity rate corrections, and corrections to the 18.6-year nutation.

In addition, the amplitude and phase of a free core nutation (FCN) assumed constant over the time span are estimated. The consideration of this component does not influence the estimation of the lower frequency components, but it allows a more realistic estimation of the residual noise in the comparison.

The estimations of precession and obliquity rate corrections from the four data sets under study are listed in Table 5. As already noted by Dehant et al. (2003) and Feissel-Vernier et al. (2005), the impact of the source selection, i.e. $c n e$ vs. $c n 7$ or $c n h$ vs. $c n 8$, is at the level of $40-50 \mu \mathrm{as} /$ year in precession, while it is small for the obliquity rate. Compared to that effect, the impact of the terrestrial frame status, i.e. $c n e$ vs. $c n h$ or $c n 7$ vs. $c n 8$ ( $2 \mu \mathrm{as})$, is barely significant.

The estimations of the 18.6-year nutation corrections are listed in Table 6. The same remarks apply: while the impact of the source selection is quite large (up to $200 \mu$ as in $\Delta \psi$ ), the impact of the terrestrial frame status $(10-20 \mu \mathrm{as})$ is statistically insignificant.
Table 6. Estimation of the 18.6-year nutation term: role of the terrestrial reference frame status and of the source selection.

\begin{tabular}{crccc}
\hline \hline Source & \multicolumn{2}{c}{$\Delta \psi(\mu$ as) } & \multicolumn{2}{c}{$\Delta \epsilon(\mu$ as $)$} \\
selection & \multicolumn{1}{c}{$\sin$} & \multicolumn{4}{c}{$\cos$} & $\sin$ & $\cos$ \\
\hline TRF approach \\
Proposed (cne) & $252 \pm 23$ & $150 \pm 26$ & $-12 \pm 9$ & $19 \pm 10$ \\
Convent. (cn7) & $80 \pm 20$ & $163 \pm 24$ & $-46 \pm 8$ & $31 \pm 10$ \\
& & & \\
Proposed (cnh) & $266 \pm 22$ & $135 \pm 25$ & $-6 \pm 8$ & $19 \pm 9$ \\
Convent. (cn8) & $71 \pm 19$ & $139 \pm 23$ & $-47 \pm 7$ & $39 \pm 9$ \\
\hline
\end{tabular}

Table 7. Agreement of the IAU2000 Precession-Nutation model, corrected for a bias and the terms listed in Tables 5 and 6, with VLBI results derived with various analysis approaches. Unit: $\mu$ as.

\begin{tabular}{|c|c|c|c|c|c|c|}
\hline \multirow[t]{2}{*}{$\begin{array}{l}\text { Reference } \\
\text { frame }\end{array}$} & \multicolumn{3}{|c|}{$\begin{array}{c}\text { TRF approach } \\
\text { Standard Dev. }(\mu \text { as })\end{array}$} & \multicolumn{3}{|c|}{$\begin{array}{c}\text { CRF approach } \\
\text { Standard Dev. ( } \mu \text { as) }\end{array}$} \\
\hline & & $\Delta \psi \sin \epsilon_{0}$ & $\Delta \epsilon$ & & $\Delta \psi \sin \epsilon_{0}$ & $\Delta \epsilon$ \\
\hline Proposed & cne & 254 & 239 & cnh & 244 & 229 \\
\hline Convent. & $\operatorname{cn} 7$ & 236 & 239 & cn8 & 228 & 233 \\
\hline
\end{tabular}

\subsection{Impact on the residual noise in celestial frame orientation}

Statistics of the residual noise in the comparisons of VLBI series of $\Delta \psi$ and $\Delta \epsilon$ with the IAU2000 model are shown in Table 7. Compared to the CRF-type solutions, the level of the residual noise of the TRF-type solutions is larger by less than 5\%. We may therefore consider that the difference in treating the station coordinates does indeed only slightly affect the high frequency noise of the nutation determinations. Note that the marginally higher noise in the analyses where the coordinates of unstable sources are not arc parameters (cne and $c n h$ ) may reflect the fact that in these analyses, the FCN is found to be time-varying (Feissel-Vernier et al. 2005), and thus consideration of a constant FCN over the data span is less appropriate.

\section{Conclusion}

The ICRF was defined on the basis of the pre-1995 astrogeodetic VLBI observations. Since then, the core of the defining sources was kept fixed in composition and coordinates, and the two subsequent updates were limited to improving non-defining source positions and extending the list of sources with coordinates referred to the original ICRF. The authors of these extensions (Fey et al. 2004) note some coordinate discrepancies with the original ICRF, but the decision to revise the whole ICRF has not been proposed to the IAU yet. The IAU decision would be based on various criteria, such as the expected accuracy gain, the impact on users, and possible inconveniences.

A number of tests and computational experiments were performed to evaluate alternative components of analysis strategies applicable to a future revision of the ICRF. The studies cited in the introduction have all shown that with the situation of astrogeodetic VLBI observations to date it is advisable to define the celestial reference frame on the basis of the post-1989 observations, and to use selected stable sources for the definition of the axes and the realisation of the primary frame (source coordinates treated as global in the data analysis). 
Using four test celestial reference frames, based on the 1980-2002 VLBI observations, and their 1984-2002 associated series of celestial pole offsets, obtained in parallel with different strategies, we have shown in addition that consideration of the global problem, i.e. the simultaneous analysis of the source position, station motion and Earth Orientation Parameters has no negative impact on the derived celestial frame or on precession and nutation measurements. Therefore we recommend that the next revision of the ICRF include the TRF approach in the analysis strategy. Note that the observations before 1990 represent less than $10 \%$ of the total available.

We conclude that the set of astro-geodetic VLBI observations accumulated since 1990 fulfils the conditions needed to provide the most precise celestial reference frame and, at the same time, to ensure the unification of the terrestrial and reference frames at the sub-millimetre level on the surface of the Earth.

\section{References}

Arias, E. F., \& Bouquillon, S. 2004, A\&A, 422, 1105

Dehant, V., Feissel-Vernier, M., de Viron, O., et al. 2003, JGRB, 108, 2275

Feissel-Vernier, M. 2003, A\&A, 403, 105

Feissel-Vernier, M. 2006, IERS Technical Note No. 34, ed. J. Souchay, \& M. Feissel-Vernier, 49

Feissel-Vernier, M., Ma, C., Gontier, A.-M., \& Barache, C. 2005, A\&A, 438, 1141

Fey, A. L., \& Charlot, P. 2000, ApJS, 128, 17

Fey, A. L., Ma, C., Arias, E. F., et al. 2004, AJ, 127, 3587

Gontier, A.-M., \& Feissel-Vernier, M. 2003, Proc. 25th meeting of the IAU, Joint Discussion 16, The International Celestial Reference System: Maintenance and Future Realization, 22 July 2003, Sydney, Australia

ICRS Product Center 2003, http://hpiers.obspm. fr/icrs-pc/analysis/ comp-CRF-2002.html

IERS 2004, http://hpiers.obspm. fr/icrs-pc/icrf/icrfdico.html

Ma, C., Arias, E. F., Eubanks, T. M., et al. 1998, AJ, 116, 516

Mathews, P. M., Herring, T. A., \& Buffett, B. A. 2002, JGRB, 107, 1029

McMillan, D. S., \& Ma, C. 1997, GRL, 24, 453 
M. Feissel-Vernier et al.: Features of a strategy for the maintenance of ICRF, Online Material $p 1$

\section{Online Material}


M. Feissel-Vernier et al.: Features of a strategy for the maintenance of ICRF, Online Material p 2

Table 8. 247 sources considered suitable for the maintenance of the ICRF.

\begin{tabular}{|c|c|c|c|c|c|c|c|c|c|}
\hline \multirow{2}{*}{$\begin{array}{c}\text { IERS } \\
\text { Des. } \\
(1)\end{array}$} & \multirow{2}{*}{$\begin{array}{l}\mathrm{Tp} \\
\text { (2) }\end{array}$} & \multirow{2}{*}{$\begin{array}{l}Z \\
(3)\end{array}$} & \multicolumn{2}{|c|}{ Flux } & \multirow{2}{*}{$\begin{array}{l}\text { Sp. } \\
\text { Ind }\end{array}$} & \multicolumn{2}{|c|}{ Struct. } & \multirow{2}{*}{$\begin{array}{c}B \\
\mathrm{Mag}\end{array}$} & \multirow{2}{*}{$\begin{array}{l}\text { Set } \\
\text { (6) }\end{array}$} \\
\hline & & & $6 \mathrm{~cm}$ & $\begin{array}{c}15 \mathrm{~cm} \\
\text { (4) }\end{array}$ & & & $\mathrm{S}$ & & \\
\hline $0003-066$ & $\mathrm{~L}$ & .347 & 1.58 & 1.69 & & 3 & 1 & 18.5 & 1 \\
\hline $0003+380$ & G & .229 & .50 & .60 & -.3 & 3 & 1 & 19.4 & 1 \\
\hline $0008-264$ & Q & 1.093 & .81 & .58 & .3 & & & 18.9 & 3 \\
\hline $0010+405$ & G & .256 & 1.05 & $1.18 *$ & -.3 & 2 & 1 & 17.9 & 2 \\
\hline $0013-005$ & Q & 1.574 & .79 & $.82 *$ & -.4 & 2 & 1 & 20.3 & 3 \\
\hline $0016+731$ & $\mathrm{Q}$ & 1.781 & 1.65 & 1.50 & .2 & 2 & 1 & 19.0 & 1 \\
\hline $0035+413$ & Q & 1.353 & .64 & $.40^{*}$ & & & & 19.9 & 2 \\
\hline $0048-097$ & $\mathrm{~L}$ & & 1.92 & 1.30 & .5 & 1 & 1 & 17.4 & 1 \\
\hline $0104-408$ & Q & .584 & .85 & $.57 *$ & .6 & & & 18.2 & 1 \\
\hline $0106+013$ & Q & 2.107 & 3.67 & 3.90 & .5 & 2 & 1 & 18.4 & 1 \\
\hline $0109+224$ & $\mathrm{~L}$ & & .78 & $.38^{*}$ & & 1 & 1 & 15.7 & 1 \\
\hline $0111+021$ & A & .047 & & & .2 & 3 & 1 & 16.3 & 1 \\
\hline $0119+041$ & Q & .637 & 1.24 & .90 & .2 & 2 & 1 & 19.5 & 1 \\
\hline $0119+115$ & Q & .570 & 1.00 & 1.80 & .3 & 2 & 1 & 19.7 & 1 \\
\hline $0133+476$ & Q & .859 & 3.26 & 2.10 & .5 & 2 & 1 & 19.5 & 1 \\
\hline $0149+218$ & $\mathrm{Q}$ & 1.320 & 1.08 & 1.40 & -.2 & 2 & 2 & 19.4 & 1 \\
\hline $0159+723$ & $\mathrm{~L}$ & & .33 & $.32 *$ & .2 & 1 & 1 & 19.2 & 1 \\
\hline $0201+113$ & Q & 3.610 & 1.20 & 1.30 & & 2 & 1 & 19.5 & 1 \\
\hline $0202+319$ & Q & 1.466 & 1.02 & 1.60 & .6 & 2 & 2 & 17.4 & 1 \\
\hline $0208-512$ & $\mathrm{~L}$ & 1.003 & 3.21 & $3.56^{*}$ & -.2 & & & 16.9 & 3 \\
\hline $0215+015$ & Q & 1.715 & .36 & $.36^{*}$ & & 1 & 1 & 16.1 & 1 \\
\hline $0219+428$ & $\mathrm{~L}$ & .444 & 1.04 & $1.14 *$ & & 2 & 1 & 15.2 & 2 \\
\hline $0221+067$ & G & .511 & .99 & 1.40 & & 2 & 2 & 20.7 & 1 \\
\hline $0224+671$ & Q & & & 1.20 & .3 & 2 & 1 & 19.5 & 1 \\
\hline $0235+164$ & $\mathrm{Q}$ & .940 & 2.79 & 2.00 & .6 & 1 & 1 & 15.5 & 1 \\
\hline $0237-233$ & $\mathrm{Q}$ & 2.225 & 3.16 & $5.34 *$ & -.7 & 4 & 3 & 16.6 & 1 \\
\hline $0256+075$ & Q & .893 & .98 & .70 & .6 & 2 & 1 & 19.0 & 1 \\
\hline $0300+470$ & $\mathrm{~L}$ & & 2.22 & $1.81 *$ & & 2 & 1 & 16.9 & 1 \\
\hline $0306+102$ & Q & .863 & .70 & 1.30 & .4 & 3 & 1 & 18.4 & 1 \\
\hline $0308-611$ & Q & & & & .7 & & & 18.5 & 1 \\
\hline $0309+411$ & $\mathrm{G}$ & .136 & .46 & .60 & .1 & 2 & 2 & 18.0 & 1 \\
\hline $0317+188$ & G & & & & -.1 & 2 & 1 & 19.0 & 1 \\
\hline $0319+121$ & Q & 2.670 & 1.54 & 1.40 & -.5 & 4 & 3 & 18.3 & 1 \\
\hline $0333+321$ & $\mathrm{Q}$ & 1.259 & 1.95 & 2.30 & & 3 & 3 & 17.5 & 1 \\
\hline $0336-019$ & Q & .852 & 2.59 & 2.70 & -.4 & 2 & 1 & 18.4 & 1 \\
\hline $0355+508$ & & & & 6.80 & & 2 & 3 & & 1 \\
\hline $0400+258$ & Q & 2.109 & 1.79 & 1.40 & & 3 & 2 & 18.0 & 1 \\
\hline $0400-319$ & Q & 1.288 & .85 & 1.15 & -.1 & & & 20.1 & 2 \\
\hline $0402-362$ & Q & 1.417 & 1.85 & $1.04 *$ & .5 & & & 17.2 & 1 \\
\hline $0406+121$ & $\mathrm{~L}$ & 1.020 & 1.62 & $1.16^{*}$ & .5 & 2 & 1 & 20.2 & 1 \\
\hline $0420-014$ & $\mathrm{Q}$ & .915 & 1.58 & 1.00 & .3 & 3 & 1 & 17.0 & 3 \\
\hline $0420+417$ & Q & & & 1.70 & & 4 & 2 & & 1 \\
\hline $0422-380$ & Q & .782 & .81 & $.49 *$ & & & & 18.1 & 3 \\
\hline $0430+052$ & G & .033 & 5.09 & 6.40 & 1.8 & 4 & 3 & 15.1 & 3 \\
\hline $0430+289$ & $\mathrm{~L}$ & & .48 & $.37 *$ & & & & 17.8 & 1 \\
\hline $0451-282$ & Q & 2.560 & 2.17 & 2.30 & .1 & & & 18.2 & 3 \\
\hline $0454-234$ & Q & 1.003 & 1.86 & $1.76^{*}$ & & 2 & 1 & 18.9 & 1 \\
\hline $0454+844$ & L & $>1.340$ & 1.40 & 1.30 & .3 & 2 & 1 & 16.5 & 1 \\
\hline $0457+024$ & $\mathrm{Q}$ & 2.384 & 1.21 & 1.30 & -.2 & 4 & 1 & 18.0 & 1 \\
\hline $0502+049$ & Q & .954 & 1.01 & .60 & .5 & 3 & 2 & 18.9 & 1 \\
\hline $0507+179$ & G & .416 & .80 & .70 & & 2 & 2 & 20.0 & 1 \\
\hline $0521-365$ & G & .055 & 8.89 & 11.50 & -.5 & & & 14.6 & 3 \\
\hline $0530-727$ & & & & & & & & & 1 \\
\hline $0537-441$ & Q & .896 & 3.96 & $3.84 *$ & .1 & 3 & 1 & 16.5 & 1 \\
\hline $0552+398$ & Q & 2.363 & 5.42 & 3.40 & & 2 & 1 & 18.3 & 1 \\
\hline $0600+177$ & & & & & & 2 & 1 & & 1 \\
\hline $0605-085$ & Q & .872 & 2.73 & $3.05^{*}$ & .2 & 3 & 1 & 17.6 & 1 \\
\hline $0607-157$ & Q & .324 & 3.13 & 1.00 & -.4 & 2 & 2 & 18.0 & 1 \\
\hline $0615+820$ & Q & .710 & 1.00 & 1.30 & & 3 & 1 & 18.9 & 1 \\
\hline $0636+680$ & Q & 3.177 & .54 & $.32 *$ & .8 & 1 & 1 & 16.6 & 1 \\
\hline $0637-752$ & $\mathrm{G}$ & .651 & 6.19 & $4.51 *$ & -.1 & & & 15.8 & 1 \\
\hline $0642+449$ & Q & 3.408 & .78 & 1.20 & -.1 & 1 & 1 & 18.5 & 1 \\
\hline $0648-165$ & & & & & & 2 & 1 & & 3 \\
\hline $0657+172$ & & & & & & 2 & 1 & & 1 \\
\hline
\end{tabular}


M. Feissel-Vernier et al.: Features of a strategy for the maintenance of ICRF, Online Material p 3

Table 8. continued.

\begin{tabular}{|c|c|c|c|c|c|c|c|c|c|}
\hline \multirow{2}{*}{$\begin{array}{c}\text { IERS } \\
\text { Des. } \\
(1)\end{array}$} & \multirow{2}{*}{$\begin{array}{l}\text { Tp } \\
\text { (2) }\end{array}$} & \multirow{2}{*}{$\begin{array}{l}Z \\
(3)\end{array}$} & \multicolumn{2}{|c|}{ Flux } & \multirow{2}{*}{$\begin{array}{l}\text { Sp. } \\
\text { Ind }\end{array}$} & \multicolumn{2}{|c|}{ Struct. } & \multirow{2}{*}{$\begin{array}{c}B \\
\mathrm{Mag}\end{array}$} & \multirow{2}{*}{$\begin{array}{l}\text { Set } \\
\text { (6) }\end{array}$} \\
\hline & & & $6 \mathrm{~cm}$ & $\begin{array}{c}15 \mathrm{~cm} \\
\text { (4) }\end{array}$ & & & S & & \\
\hline $0707+476$ & $\mathrm{Q}$ & 1.292 & 1.00 & .80 & -.3 & 2 & 1 & 18.2 & 1 \\
\hline $0716+714$ & $\mathrm{~L}$ & & 1.12 & .70 & .4 & 1 & 1 & 15.5 & 1 \\
\hline $0718+792$ & & & & 1.00 & & 2 & 1 & & 1 \\
\hline $0722+145$ & & & & 5.30 & & 2 & 2 & & 2 \\
\hline $0727-115$ & Q & & & & .1 & 1 & 1 & & 1 \\
\hline $0735+178$ & $\mathrm{~L}$ & $>.424$ & 1.99 & 2.00 & .1 & 3 & 1 & 16.2 & 3 \\
\hline $0736+017$ & $\mathrm{Q}$ & .191 & 1.92 & 2.90 & .1 & 3 & 3 & 16.5 & 1 \\
\hline $0743-006$ & $\mathrm{Q}$ & .994 & 1.31 & $1.01 *$ & .4 & 2 & 1 & 18.1 & 3 \\
\hline $0748+126$ & $\mathrm{Q}$ & .889 & 2.22 & 2.00 & .5 & 2 & 2 & 18.7 & 1 \\
\hline $0754+100$ & L & .280 & 1.48 & $1.04 *$ & .2 & 2 & 1 & 15.0 & 1 \\
\hline $0804+499$ & $\mathrm{Q}$ & 1.432 & 2.07 & 1.30 & .5 & 2 & 1 & 18.3 & 1 \\
\hline $0805+410$ & $\mathrm{Q}$ & 1.420 & .77 & .70 & -.3 & 2 & 1 & 19.0 & 1 \\
\hline $0808+019$ & $\mathrm{~L}$ & & .67 & .39 & .4 & 1 & 1 & 17.2 & 3 \\
\hline $0812+367$ & Q & 1.025 & 1.01 & 1.20 & & 2 & 1 & 19.0 & 2 \\
\hline $0818-128$ & $\mathrm{~L}$ & & .86 & .90 & -.1 & 3 & 1 & 15.0 & 1 \\
\hline $0821+394$ & $\mathrm{Q}$ & 1.216 & .99 & 1.90 & -.2 & 2 & 2 & 18.3 & 1 \\
\hline $0823+033$ & $\mathrm{~L}$ & .506 & 1.43 & 1.42 & .9 & 2 & 1 & 16.8 & 1 \\
\hline $0827+243$ & $\mathrm{Q}$ & .941 & .89 & 1.30 & & 2 & 2 & 17.3 & 1 \\
\hline $0828+493$ & $\mathrm{~L}$ & .548 & 1.02 & .50 & -.3 & 2 & 1 & 18.8 & 1 \\
\hline $0829+046$ & $\mathrm{~L}$ & .180 & .70 & .70 & .2 & 3 & 2 & 16.4 & 3 \\
\hline $0836+710$ & Q & 2.218 & 2.57 & 4.40 & -.3 & 3 & 3 & 17.3 & 2 \\
\hline $0850+581$ & Q & 1.322 & 1.41 & 1.60 & .8 & 2 & 2 & 18.3 & 2 \\
\hline $0851+202$ & $\mathrm{~L}$ & .306 & 2.61 & $3.38 *$ & -.4 & 2 & 1 & 15.4 & 1 \\
\hline $0859-140$ & $\mathrm{Q}$ & 1.339 & 2.25 & 2.90 & -.4 & 3 & 3 & 16.6 & 1 \\
\hline $0859+470$ & $\mathrm{Q}$ & 1.462 & 1.78 & 2.20 & -.1 & 3 & 2 & 19.2 & 2 \\
\hline $0912+029$ & G & .427 & .89 & .68 & -.3 & 2 & 1 & 19.6 & 2 \\
\hline $0917+624$ & $\mathrm{Q}$ & 1.446 & 1.24 & 1.60 & .2 & 2 & 1 & 19.5 & 1 \\
\hline $0919-260$ & Q & 2.300 & 2.38 & 1.20 & .2 & 3 & 2 & 18.4 & 1 \\
\hline $0920+390$ & & & & & & & & & 3 \\
\hline $0920-397$ & Q & .591 & 1.51 & 2.10 & -.2 & & & 18.4 & 3 \\
\hline $0925-203$ & G & .348 & .69 & $.81 *$ & -.2 & & & 16.4 & 1 \\
\hline $0945+408$ & $\mathrm{Q}$ & 1.252 & 1.80 & 1.70 & & 2 & 2 & 18.0 & 1 \\
\hline $0951+693$ & G & & .09 & .50 & & & & 6.9 & 1 \\
\hline $0952+179$ & Q & 1.478 & .74 & 1.00 & -.3 & 3 & 3 & 17.2 & 1 \\
\hline $0954+658$ & L & .367 & 1.46 & .90 & .4 & 2 & 1 & 16.7 & 1 \\
\hline $0955+326$ & G & .530 & .71 & .70 & -.3 & 2 & 2 & 15.8 & 1 \\
\hline $1004+141$ & Q & 2.707 & .74 & .81 & -.1 & 3 & 2 & 18.4 & 1 \\
\hline $1012+232$ & G & .565 & 1.09 & $.74 *$ & -.3 & 2 & 1 & 17.8 & 1 \\
\hline $1020+400$ & Q & 1.254 & .87 & 1.20 & -.3 & 3 & 1 & 17.4 & 3 \\
\hline $1022+194$ & Q & .828 & .60 & 1.04 & .7 & 2 & 2 & 17.5 & 3 \\
\hline $1034-293$ & Q & .312 & 1.51 & 1.30 & .2 & 1 & 1 & 16.5 & 1 \\
\hline $1038+528$ & Q & .677 & .70 & $.43 *$ & .2 & & & 16.9 & 1 \\
\hline $1039+811$ & Q & 1.260 & 1.14 & $.90 *$ & .4 & 2 & 1 & 17.9 & 1 \\
\hline $1044+719$ & $\mathrm{Q}$ & 1.150 & .71 & 1.00 & .1 & 1 & 1 & 19.0 & 1 \\
\hline $1049+215$ & $\mathrm{Q}$ & 1.300 & 1.08 & 1.70 & -.4 & 2 & 1 & 19.0 & 3 \\
\hline $1053+815$ & $\mathrm{Q}$ & .706 & .77 & .60 & -.4 & 1 & 1 & 20.0 & 1 \\
\hline $1055+018$ & $\mathrm{Q}$ & .888 & 3.38 & 2.87 & & 2 & 2 & 18.3 & 1 \\
\hline $1057-797$ & $\mathrm{Q}$ & & & & .6 & & & 19.3 & 1 \\
\hline $1101+384$ & $\mathrm{~L}$ & .031 & .70 & 1.00 & -.1 & 1 & 1 & 12.9 & 1 \\
\hline $1111+149$ & Q & .869 & .49 & $.55^{*}$ & .4 & 2 & 1 & 17.9 & 1 \\
\hline $1116+128$ & Q & 2.118 & 1.87 & 1.74 & -.3 & 3 & 2 & 19.3 & 3 \\
\hline $1123+264$ & Q & 2.341 & .83 & .70 & .8 & 2 & 1 & 18.0 & 1 \\
\hline $1124-186$ & Q & 1.048 & 1.62 & .60 & .5 & 1 & 1 & 18.6 & 1 \\
\hline $1128+385$ & $\mathrm{Q}$ & 1.733 & .77 & .90 & -.2 & 1 & 1 & 19.5 & 1 \\
\hline $1130+009$ & Q & & & .33 & & 2 & 1 & 19.0 & 1 \\
\hline $1144-379$ & Q & 1.048 & 2.22 & $1.07 *$ & -.2 & & & 16.2 & 3 \\
\hline $1144+402$ & $\mathrm{Q}$ & 1.089 & 1.03 & .90 & -.2 & 1 & 1 & 18.0 & 1 \\
\hline $1145-071$ & Q & 1.342 & 1.21 & .97 & .2 & 3 & 1 & 18.7 & 1 \\
\hline $1150+497$ & $\mathrm{Q}$ & .334 & .72 & 1.60 & -.2 & 2 & 1 & 17.1 & 2 \\
\hline $1150+812$ & Q & 1.250 & 1.18 & 1.20 & -.1 & 2 & 2 & 19.4 & 1 \\
\hline $1156+295$ & Q & .729 & 1.46 & 1.30 & & 2 & 2 & 14.4 & 1 \\
\hline $1213-172$ & $\mathrm{G}$ & & & 1.20 & -.1 & 1 & 1 & 21.4 & 3 \\
\hline
\end{tabular}


M. Feissel-Vernier et al.: Features of a strategy for the maintenance of ICRF, Online Material p 4

Table 8. continued.

\begin{tabular}{|c|c|c|c|c|c|c|c|c|c|}
\hline \multirow{2}{*}{$\begin{array}{c}\text { IERS } \\
\text { Des. } \\
(1)\end{array}$} & \multirow{2}{*}{$\begin{array}{l}\mathrm{Tp} \\
(2)\end{array}$} & \multirow{2}{*}{$\begin{array}{l}Z \\
(3)\end{array}$} & \multicolumn{2}{|c|}{$\begin{array}{ll}\text { Flux } \\
\end{array}$} & \multirow{2}{*}{$\begin{array}{l}\text { Sp. } \\
\text { Ind }\end{array}$} & \multicolumn{2}{|c|}{ Struct. } & \multirow{2}{*}{$\begin{array}{c}B \\
\text { Mag }\end{array}$} & \multirow{2}{*}{$\begin{array}{l}\overline{\text { Set }} \\
\text { (6) }\end{array}$} \\
\hline & & & $6 \mathrm{~cm}$ & $\begin{array}{c}15 \mathrm{~cm} \\
\text { (4) }\end{array}$ & & & $S$ & & \\
\hline $1216+487$ & $\mathrm{Q}$ & 1.076 & 1.08 & .70 & .2 & 2 & 1 & 18.6 & 1 \\
\hline $1219+044$ & $\mathrm{Q}$ & .965 & 1.35 & .65 & -1.2 & 2 & 1 & 18.0 & 1 \\
\hline $1219+285$ & $\mathrm{~L}$ & .102 & .72 & 1.47 & & 3 & 2 & 16.1 & 1 \\
\hline $1221+809$ & $\mathrm{~L}$ & & .52 & $.40^{*}$ & -.3 & 2 & 1 & 19.0 & 1 \\
\hline $1222+131$ & G & .003 & 3.17 & 5.20 & -.5 & & & 12.3 & 3 \\
\hline $1228+126$ & G & .004 & 71.90 & & -.6 & 3 & 3 & 12.9 & 1 \\
\hline $1237-101$ & $\mathrm{Q}$ & .750 & 1.53 & 1.63 & -.2 & & & 18.1 & 3 \\
\hline $1244-255$ & $\mathrm{Q}$ & .638 & 2.32 & 1.36 & .2 & & & 17.4 & 1 \\
\hline $1252+119$ & Q & .870 & .72 & 1.80 & -.2 & 2 & 1 & 16.6 & 3 \\
\hline $1300+580$ & $\mathrm{Q}$ & & & & -.5 & 1 & 1 & & 1 \\
\hline $1308+326$ & Q & .997 & 1.50 & $1.57 *$ & & 1 & 1 & 15.2 & 1 \\
\hline $1308+328$ & Q & 1.650 & 1.08 & & & & & 17.7 & 3 \\
\hline $1313-333$ & $\mathrm{Q}$ & 1.210 & 1.09 & 1.23 & .5 & 1 & 1 & 18.7 & 1 \\
\hline $1315+346$ & $\mathrm{Q}$ & 1.050 & .34 & $.54 *$ & -.1 & 2 & 1 & 18.7 & 1 \\
\hline $1324+224$ & $\mathrm{Q}$ & 1.400 & .65 & & & 1 & 1 & 18.9 & 2 \\
\hline $1334-127$ & Q & .539 & 2.84 & 1.94 & .2 & 2 & 1 & 19.0 & 1 \\
\hline $1342+662$ & $\mathrm{Q}$ & .766 & .54 & $.51 *$ & .1 & 2 & 1 & 19.7 & 2 \\
\hline $1342+663$ & Q & 1.351 & .82 & $.61 *$ & .5 & 2 & 1 & 19.3 & 1 \\
\hline $1347+539$ & $\mathrm{Q}$ & .976 & .96 & .90 & -.1 & 3 & 2 & 17.4 & 3 \\
\hline $1349-439$ & $\mathrm{~L}$ & .053 & .76 & $.54 *$ & .6 & & & 16.4 & 1 \\
\hline $1351-018$ & $\mathrm{Q}$ & 3.707 & .82 & .84 & -.1 & 1 & 1 & 19.3 & 1 \\
\hline $1354-152$ & $\mathrm{Q}$ & 1.890 & .84 & 1.17 & .1 & 1 & 1 & 18.1 & 3 \\
\hline $1354+195$ & G & .719 & 2.62 & 1.80 & -.1 & 3 & 2 & 16.0 & 1 \\
\hline $1357+769$ & Q & & & & .7 & 1 & 1 & 19.0 & 1 \\
\hline $1402+044$ & $\mathrm{Q}$ & 3.211 & .71 & .65 & .1 & 2 & 1 & 19.0 & 1 \\
\hline $1406-076$ & Q & 1.493 & .82 & 1.30 & .2 & 2 & 1 & 19.6 & 1 \\
\hline $1413+135$ & $\mathrm{~L}$ & .247 & 1.20 & .90 & -.1 & 1 & 3 & 20.5 & 1 \\
\hline $1417+273$ & & & & & & 2 & 1 & & 2 \\
\hline $1417+385$ & $\mathrm{Q}$ & 1.832 & .87 & $.51 *$ & & & & 19.3 & 3 \\
\hline $1418+546$ & $\mathrm{~L}$ & .152 & 1.09 & 1.00 & .2 & 2 & 2 & 15.7 & 1 \\
\hline $1424-418$ & Q & 1.522 & 2.17 & 2.22 & -.4 & & & 18.9 & 1 \\
\hline $1432+200$ & & & & & & 2 & 1 & & 2 \\
\hline $1442+101$ & Q & 3.535 & 1.15 & 2.01 & -.6 & 3 & 3 & 17.8 & 1 \\
\hline $1448+762$ & G & .899 & .68 & 1.00 & .5 & 1 & 1 & 22.3 & 3 \\
\hline $1451-375$ & G & .314 & 1.84 & 1.40 & .4 & & & 16.7 & 1 \\
\hline $1451-400$ & $\mathrm{Q}$ & 1.810 & .61 & $.71 *$ & -.2 & & & 18.7 & 1 \\
\hline $1458+718$ & G & .904 & 3.76 & 6.90 & -.5 & 3 & 3 & 16.8 & 3 \\
\hline $1459+480$ & & & & .50 & & 2 & 1 & 17.1 & 1 \\
\hline $1504-166$ & $\mathrm{Q}$ & .876 & 2.84 & 2.20 & -.4 & 3 & 1 & 18.5 & 1 \\
\hline $1504+377$ & G & .674 & 1.10 & 1.10 & .2 & 2 & 1 & 21.2 & 2 \\
\hline $1510-089$ & Q & .360 & 4.36 & 3.14 & .3 & 3 & 1 & 16.5 & 1 \\
\hline $1514+197$ & $\mathrm{~L}$ & 1.070 & .50 & .50 & & 2 & 1 & 18.7 & 1 \\
\hline $1514-241$ & $\mathrm{~L}$ & .048 & 1.94 & 1.90 & -.1 & & & 14.8 & 1 \\
\hline $1519-273$ & $\mathrm{~L}$ & & 1.83 & .99 & .2 & & & 17.7 & 1 \\
\hline $1538+149$ & $\mathrm{Q}$ & .605 & 1.95 & 1.50 & & 2 & 1 & 17.3 & 1 \\
\hline $1546+027$ & Q & .412 & 1.11 & .79 & .2 & 2 & 1 & 17.8 & 1 \\
\hline $1547+507$ & Q & 2.169 & .74 & $.69 *$ & .1 & 3 & 3 & 18.4 & 1 \\
\hline $1555+001$ & Q & 1.772 & 2.18 & .58 & .2 & 1 & 1 & 20.7 & 3 \\
\hline $1557+032$ & Q & 3.891 & .48 & $.46^{*}$ & & & & 19.8 & 3 \\
\hline $1600+335$ & & & & 2.70 & & 3 & 1 & 23.2 & 1 \\
\hline $1610-771$ & Q & 1.710 & 5.55 & $3.80 *$ & .8 & & & 19.2 & 1 \\
\hline $1614+051$ & Q & 3.217 & .92 & .63 & .4 & 2 & 1 & 19.6 & 1 \\
\hline $1616+063$ & Q & 2.088 & .89 & 1.10 & -.1 & 2 & 1 & 19.6 & 2 \\
\hline $1622-253$ & Q & .786 & 2.20 & $2.27 *$ & -.1 & 1 & 1 & 20.6 & 1 \\
\hline $1622-297$ & Q & .815 & 1.86 & 2.20 & .2 & & & 19.5 & 1 \\
\hline $1624+416$ & Q & 2.550 & 1.58 & 1.50 & -.3 & 3 & 2 & 22.0 & 1 \\
\hline $1633+382$ & Q & 1.807 & 4.08 & 2.10 & .8 & 3 & 1 & 17.7 & 1 \\
\hline $1637+574$ & $\mathrm{G}$ & .751 & 1.75 & 1.40 & .6 & 2 & 1 & 16.9 & 1 \\
\hline $1639+230$ & & & & & & & & & 2 \\
\hline $1641+399$ & Q & .594 & 5.65 & 7.60 & & 4 & 1 & 16.0 & 3 \\
\hline
\end{tabular}


M. Feissel-Vernier et al.: Features of a strategy for the maintenance of ICRF, Online Material p 5

Table 8. continued.

\begin{tabular}{|c|c|c|c|c|c|c|c|c|c|}
\hline \multirow{2}{*}{$\begin{array}{c}\text { IERS } \\
\text { Des. } \\
(1)\end{array}$} & \multirow{2}{*}{$\begin{array}{l}\mathrm{Tp} \\
\text { (2) }\end{array}$} & \multirow{2}{*}{$\begin{array}{l}Z \\
(3)\end{array}$} & \multicolumn{2}{|c|}{ Flux } & \multirow{2}{*}{$\begin{array}{l}\text { Sp. } \\
\text { Ind }\end{array}$} & \multicolumn{2}{|c|}{ Struct. } & \multirow{2}{*}{$\begin{array}{c}B \\
\text { Mag }\end{array}$} & \multirow{2}{*}{$\begin{array}{l}\text { Set } \\
\text { (6) }\end{array}$} \\
\hline & & & $6 \mathrm{~cm}$ & $\begin{array}{c}15 \mathrm{~cm} \\
\text { (4) }\end{array}$ & & & S & & \\
\hline $1652+398$ & $\mathrm{~L}$ & .033 & 1.31 & $1.45^{*}$ & & 3 & 2 & 13.8 & 1 \\
\hline $1655+077$ & $\mathrm{Q}$ & .621 & 1.60 & 1.63 & .4 & 3 & 3 & 20.0 & 1 \\
\hline $1656+053$ & $\mathrm{Q}$ & .879 & 1.38 & 1.61 & .5 & 3 & 2 & 16.5 & 3 \\
\hline $1657-261$ & & & & 1.10 & -.2 & & & & 1 \\
\hline $1705+018$ & Q & 2.576 & .54 & $.53 *$ & .1 & 2 & 1 & 18.8 & 3 \\
\hline $1705+456$ & Q & .646 & .47 & $.66^{*}$ & & 3 & 2 & 17.3 & 3 \\
\hline $1717+178$ & $\mathrm{~L}$ & & .94 & 1.00 & .6 & 2 & 1 & 19.1 & 1 \\
\hline $1725+044$ & G & .293 & .89 & .84 & .8 & 2 & 1 & 17.0 & 1 \\
\hline $1732+389$ & Q & .976 & 1.15 & $.67 *$ & .8 & 2 & 1 & 20.6 & 1 \\
\hline $1734+363$ & G & .893 & .33 & $.32 *$ & & & & 20.9 & 2 \\
\hline $1743+173$ & Q & 1.702 & .94 & .90 & .1 & 2 & 1 & 20.8 & 1 \\
\hline $1744+557$ & & & & & & & & & 3 \\
\hline $1745+624$ & $\mathrm{Q}$ & 3.889 & .59 & $.59 *$ & & 1 & 2 & 18.8 & 1 \\
\hline $1749+096$ & $\mathrm{Q}$ & .320 & 2.46 & 1.07 & -.2 & 1 & 1 & 16.8 & 1 \\
\hline $1751+288$ & Q & & & 1.10 & & 2 & 1 & 20.0 & 1 \\
\hline $1758+388$ & $\mathrm{Q}$ & 2.092 & .92 & .60 & 1.2 & & & 18.0 & 3 \\
\hline $1800+440$ & $\mathrm{Q}$ & .663 & 1.02 & .79 & .4 & 1 & 1 & 17.9 & 3 \\
\hline $1803+784$ & Q & .680 & 2.63 & 2.60 & .3 & 2 & 1 & 15.9 & 1 \\
\hline $1807+698$ & $\mathrm{~L}$ & .050 & 1.74 & 3.10 & -.3 & 3 & 2 & 14.2 & 1 \\
\hline $1815-553$ & Q & & & & -.1 & & & 19.3 & 1 \\
\hline $1821+107$ & Q & 1.364 & 1.23 & $.95^{*}$ & .5 & 3 & 1 & 17.3 & 1 \\
\hline $1823+568$ & Q & .663 & 1.67 & 1.60 & .2 & 1 & 1 & 19.3 & 1 \\
\hline $1830+285$ & $\mathrm{Q}$ & .594 & .98 & 1.30 & & 3 & 2 & 17.2 & 1 \\
\hline $1842+681$ & $\mathrm{Q}$ & .475 & .93 & 1.20 & -.4 & 2 & 1 & 18.1 & 1 \\
\hline $1849+670$ & G & .657 & .59 & $.95^{*}$ & & 1 & 2 & 16.9 & 3 \\
\hline $1901+319$ & G & .635 & 1.81 & 3.10 & & 4 & 3 & 17.6 & 3 \\
\hline $1908-201$ & & & & 2.30 & & & & & 1 \\
\hline $1921-293$ & $\mathrm{Q}$ & .352 & 14.33 & $4.60^{*}$ & & 2 & 1 & 18.2 & 1 \\
\hline $1923+210$ & & & & & & 3 & 2 & & 1 \\
\hline $1928+738$ & G & .303 & 3.34 & 3.00 & & 3 & 2 & 16.1 & 1 \\
\hline $1933-400$ & $\mathrm{Q}$ & .965 & 1.44 & $1.20^{*}$ & .1 & & & 19.0 & 1 \\
\hline $1936-155$ & Q & 1.657 & .80 & 1.30 & .2 & 1 & 1 & 20.3 & 1 \\
\hline $1937-101$ & Q & 3.787 & .75 & .90 & -.3 & 3 & 1 & 17.0 & 1 \\
\hline $1954-388$ & $\mathrm{Q}$ & .630 & 2.02 & 1.00 & & & & 17.1 & 1 \\
\hline $1954+513$ & $\mathrm{Q}$ & 1.223 & 1.61 & 1.40 & -.1 & 2 & 1 & 18.5 & 1 \\
\hline $1958-179$ & Q & .652 & 2.70 & 1.12 & .1 & 1 & 1 & 18.6 & 1 \\
\hline $2005+642$ & $\mathrm{Q}$ & 1.574 & .72 & & & & & 19.0 & 2 \\
\hline $2017+745$ & $\mathrm{Q}$ & 2.191 & .37 & $.34 *$ & & 2 & 1 & 18.1 & 2 \\
\hline $2021+317$ & & & & & & 3 & 1 & & 1 \\
\hline $2037+511$ & Q & 1.687 & 3.79 & 5.00 & & 3 & 3 & 21.0 & 1 \\
\hline $2051+745$ & $\mathrm{~L}$ & & .53 & $.43^{*}$ & & 2 & 1 & 20.4 & 2 \\
\hline $2052-474$ & $\mathrm{Q}$ & 1.489 & 2.45 & $3.00^{*}$ & -.3 & & & 18.1 & 1 \\
\hline $2059+034$ & Q & 1.015 & 1.36 & .57 & .5 & 2 & 1 & 17.8 & 1 \\
\hline $2113+293$ & $\mathrm{Q}$ & 1.514 & 1.45 & 1.12 & & 1 & 1 & 20.6 & 3 \\
\hline $2128-123$ & G & .501 & 1.99 & 1.69 & .1 & 3 & 2 & 16.1 & 1 \\
\hline $2134+004$ & Q & 1.932 & 11.49 & 6.50 & .8 & 4 & 1 & 16.8 & 1 \\
\hline $2136+141$ & Q & 2.427 & 1.11 & 1.19 & & 1 & 1 & 18.9 & 1 \\
\hline $2149-307$ & $\mathrm{Q}$ & 2.345 & 1.34 & 1.35 & -.2 & & & 17.9 & 1 \\
\hline $2200+420$ & $\mathrm{~L}$ & .069 & 2.94 & 4.20 & -.5 & 3 & 1 & 14.7 & 1 \\
\hline $2201+315$ & G & 298 & 2.81 & 2.02 & .2 & 3 & 1 & 15.6 & 1 \\
\hline $2209+236$ & A & & & .70 & .2 & 1 & 1 & 19.0 & 1 \\
\hline $2216-038$ & $\mathrm{Q}$ & .901 & 1.63 & 1.62 & .7 & 3 & 1 & 16.4 & 1 \\
\hline $2230+114$ & Q & 1.037 & 3.97 & $4.93^{*}$ & -.5 & 4 & 2 & 17.3 & 1 \\
\hline $2253+417$ & $\mathrm{Q}$ & 1.476 & .99 & 1.50 & -.3 & 4 & 1 & 19.4 & 1 \\
\hline $2254+024$ & $\mathrm{Q}$ & 2.089 & .51 & $.46^{*}$ & .2 & 1 & 1 & 18.0 & 3 \\
\hline $2255-282$ & G & .927 & 2.13 & $1.38^{*}$ & .4 & 1 & 2 & 16.8 & 1 \\
\hline $2318+049$ & Q & .623 & 1.05 & $1.23^{*}$ & -.2 & 2 & 1 & 19.0 & 1 \\
\hline $2328+107$ & $\mathrm{Q}$ & 1.489 & .96 & 1.05 & -.1 & 3 & 2 & 18.5 & 3 \\
\hline $2335-027$ & $\mathrm{Q}$ & 1.072 & .65 & .65 & .2 & 3 & 1 & 19.6 & 1 \\
\hline $2351+456$ & Q & 1.986 & 1.48 & $1.41 *$ & -.1 & 3 & 2 & 20.6 & 1 \\
\hline $2355-106$ & Q & 1.622 & 1.62 & $.47 *$ & .5 & 1 & 1 & 18.9 & 1 \\
\hline
\end{tabular}

